\title{
Documentos
}

\section{MECANISMO DE ACCIÓN DEL LEVONORGESTREL EN LA ANTICONCEPCIÓN DE EMERGENCIA}

\author{
Horacio Croxatto A. ${ }^{1}$, María Elena Ortiz S. ${ }^{1, a}$ \\ ${ }^{1}$ Investigadores asociados, Facultad de Ciencias Biológicas, Pontificia Universidad Católica de Chile
}

aióloga

\section{RESUMEN}

En esta revisión se actualiza el mecanismo de acción del levonorgestrel (LNG) usado en anticoncepción de emergencia. El análisis crítico de la estimación de la eficacia anticonceptiva del LNG indica que su tasa de falla es más alta que la publicada. EI LNG aumenta la viscosidad del moco cervical impidiendo que los espermatozoides del reservorio cervical vayan a renovar la población espermática en el sitio de fecundación. Diversos autores han documentado que LNG suprime el pico preovulatorio de gonadotrofinas e interfiere con el proceso ovulatorio en la mujer y en modelos animales. Administrado después de la fecundación en rata y Cebus apella, no interfiere con la implantación del embrión. Se concluye que LNG previene el embarazo solamente cuando se administra en un momento del ciclo menstrual en el cual puede impedir la fecundación y que el método falla cuando la administración es más tardía.

\section{PALABRAS CLAVES: Levonorgestrel, migración espermática, ovulación}

\section{SUMMARY}

This review updates the mechanism of action of levonorgestrel (LNG) used for emergency contraception. A critical analysis of estimates of the contraceptive efficacy of LNG indicates that its failure rate is higher than previously reported. Under the effect of LNG, cervical mucus turns highly viscous and hinders the exit of sperm from the cervical reservoir needed to renew the sperm population at the site of fertilization. Several authors have documented that LNG suppresses the preovulatory surge of gonadotropins and interferes with the ovulatory process in women and in animal models. LNG does not interfere with embryo implantation in rats and Cebus apella, when it is administered after fertilization. In conclusion, LNG prevents pregnancy only when it is administered at a time of the menstrual cycle in which it can impede fertilization and the method fails when it is given at later stages of the cycle.

\section{KEY WORDS: Levonorgestrel, sperm migration, ovulation}

\section{INTRODUCCIÓN}

La anticoncepción hormonal de emergencia (AE) comprende píldoras anticonceptivas que las mujeres pueden utilizar en los días siguientes a una relación sexual no protegida con el fin de evitar un embarazo no deseado. La AE está indi- cada cuando la mujer que no desea un embarazo ha sido forzada a tener una relación sexual, o la tuvo voluntariamente pero sin usar protección anticonceptiva o la protección que usó fue defectuosa. Es solamente una alternativa de emergencia cuando no hay otra forma de impedir un embarazo. No es adecuada para ser usada en 
vez de la anticoncepción hormonal de uso regular ya que es mucho menos efectiva y por usar dosis más altas produce más síntomas adversos.

$\mathrm{La} A E$, comprende las píldoras de progestina sola que contienen $0,75 \mathrm{mg}$ de levonorgestrel (LNG) y las píldoras combinadas que contienen $0,5 \mathrm{mg}$ de LNG y $0,1 \mathrm{mg}$ de etinil estradiol (Método de Yuzpe). Los productos disponibles en Chile son Postinor-2 y TACE, que suministran dos tabletas de LNG que contienen $0,75 \mathrm{mg}$ cada una y que se toman dentro de las primeras 120 horas que siguen a la relación sexual, y lo antes posible para mayor eficacia (1).

EI LNG es un derivado de la 19-nortestosterona que actuando a través del receptor de la progesterona imita los efectos de la hormona natural por lo que se clasifica como agonista o progestina. Como toda progestina, mantiene el embarazo en animales ovariectomizados, convierte el endometrio proliferativo en receptivo e inhibe la ovulación cuando se administra en la fase folicular.

\section{EFICACIA ANTICONCEPTIVA DEL LNG USADO EN AE}

Hasta ahora no se ha podido determinar la eficacia anticonceptiva del LNG usado como AE con una precisión comparable a la del resto de los métodos anticonceptivos debido a que no es posible contar con un grupo control que permita establecer cuantos embarazos se producirían si no se usara el método. Para el resto de los métodos anticonceptivos los grupos controles son las parejas que intentan embarazarse teniendo relaciones sexuales ad libitum, sin usar ninguna forma de protección. Para establecer cuantos embarazos previene la $A E$ sería necesario tratar mujeres que solicitan el método con LNG o con placebo en un diseño aleatorio y doble ciego, lo cual es éticamente inadmisible. Por ello sólo hay estimaciones de su eficacia. Piaggio y cols. (2) estiman que cuando se usa LNG en los primeros 4 días que siguen a la relación sexual, se previene $79-85 \%$ de los embarazos esperados y cuando se usa en el $5^{\circ}$ día previene sólo el $31 \%$.

Los métodos usados para estimar la eficacia anticonceptiva de la AE han sido cuestionados (36) pues conducen a sobrestimar la proporción de embarazos que se previenen debido a que se presume erróneamente un mayor número de mujeres expuesta al riesgo de embarazo que el número real. En los estudios de eficacia se le atribuye a la población de usuarias de AE la misma fertilidad que a la población que estudió Wilcox y cols (7) para establecer la probabilidad de embarazo después de un coito único. La población que estudió Wilcox y cols estaba constituida mayoritariamente por parejas de fertilidad probada y todas sanas, que querían embarazarse de modo que su probabilidad de embarazo es la más alta que se puede esperar. Esa probabilidad es la que se aplica a las mujeres que acuden a solicitar $A E$, a pesar de que no son probadamente fértiles y algunas son portadoras de infecciones genitales que reducen la fertilidad. Se sabe que en la población general, $10-15 \%$ de las parejas son infértiles de modo que la fertilidad de esta población está sobrestimada. Por otra parte Senosiain y cols (8) reportaron que cerca del $40 \%$ de las mujeres que solicitan $A E$ por falla de un método de barrera no tiene espermatozoides en la vagina. Como este examen no se hace en los estudios de eficacia, estas mujeres se incluyen inadvertidamente en el grupo en riesgo de embarazarse. Todos estos factores llevan a sobrestimar la eficacia anticonceptiva de este método en una magnitud imponderable.

Si la eficacia de este método fuera vecina a $100 \%$ no habría duda alguna que tendría que evitar algunos embarazos por interferencia con eventos posteriores a la fecundación, pero no es así. De hecho una explicación coherente de su mecanismo de acción necesariamente tiene que dar cuenta no sólo de cómo evita algunos embarazos sino que también por qué no los evita todos ( 9 , 10). Esta revisión resume los resultados de investigaciones que aportan información sobre el mecanismo de acción de la AE con LNG.

\section{EFECTO DEL LNG SOBRE LA MIGRACIÓN Y VITALIDAD ESPERMÁTICA}

Un coito único puede producir un embarazo solamente si tiene lugar en el día en que ocurre la ovulación o en los 5 días que la preceden y en cualquiera de estos días la probabilidad de que ocurra un embarazo reconocible clínicamente no supera el $27 \%$ (7). Estos seis días constituyen el período fértil del ciclo menstrual y su distribución asimétrica en torno al día de la ovulación se debe a dos factores. Por una parte los espermatozoides pueden conservar su viabilidad y capacidad fecundante hasta por cinco días en las criptas del cuello uterino. Allí se forma un reservorio que surte continuamente a la trompa de Fallopio de nuevos espermatozoides durante varios días hasta que se produce la ovulación. Por otra parte, el óvulo necesita ser fecundado pocas horas después de la ovulación para generar un zigoto sano que sea 
viable mas allá de la implantación. El $83 \%$ de los días del período fértil están antes de la ovulación, y cuando el coito ocurre en alguno de estos días, los espermatozoides tienen que esperar entre $1 \mathrm{y}$ 5 días en el tracto genital de la mujer hasta que ocurra la ovulación. Esta circunstancia ofrece una posibilidad real y concreta de que en la mayoría de los casos el LNG pueda actuar sobre la migración o vitalidad de los espermatozoides o sobre el proceso ovulatorio o sobre ambos y de ese modo prevenir la fecundación.

EI LNG no tiene in vitro un efecto directo sobre los espermatozoides que afecte de un modo adverso su movilidad o capacidad fecundante al menos en las concentraciones que ocurrirían in vivo $(11,12)$. Por otra parte, diversos estudios han confirmado que el LNG actúa sobre las células mucosas del cuello uterino alterando su secreción de un modo que la torna muy viscosa hasta el punto de suprimir totalmente el avance de los espermatozoides a través del moco cervical (13, 14). Kesserü y cols (15) reportaron que la administración de 0,4 mg de LNG 3-10 horas después del coito produce una disminución del número de espermatozoides recuperados de la cavidad uterina, 3 horas después del tratamiento; aumenta el $\mathrm{pH}$ del fluido uterino a las 5 horas lo cual inmoviliza a los espermatozoides; y aumenta la viscosidad del moco cervical a partir de las 9 horas impidiendo el paso de más espermatozoides hacia la cavidad uterina. Estas observaciones fueron hechas utilizando un $57 \%$ de la dosis que se usa actualmente, no obstante son relevantes al modo de acción del LNG usado como AE.

Los pocos datos disponibles sobre migración espermática en la mujer, muestran que ocurre como en el resto de los mamíferos. Después de una fase rápida y breve en la cual los primeros espermatozoides llegan a la trompa pocos minutos después del coito, hay una fase sostenida en la cual los espermatozoides migran en sucesivas cohortes desde el reservorio cervical hacia la trompa en el curso de varios días. Los únicos experimentos que han evaluado la capacidad fecundante de estas dos poblaciones de espermatozoides revelan que sólo los de la fase sostenida tienen la capacidad de fecundar (revisión en referencia 16). La fase sostenida mantiene en la trompa una población de espermatozoides con capacidad fecundante hasta que se produzca la ovulación. Los que llegan a la trompa no se quedan en ella ni persisten viables por mucho tiempo. En efecto, algunos pasan a la cavidad peritoneal o bien se adhieren al epitelio de la trompa por algunas horas,

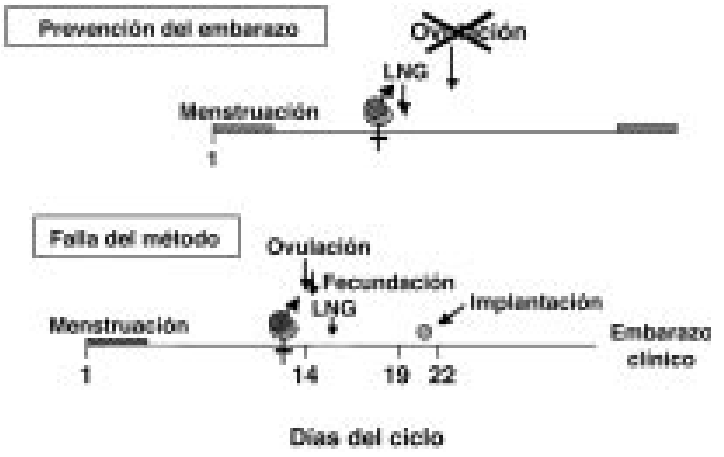

Figura 1. Se ilustra en forma esquemática la explicación de cómo levonorgestrel (LNG) usado para anticoncepción de emergencia previene algunos embarazos pero no los previene todos. En la parte superior se ilustra que después de tener una relación sexual en el período fértil del ciclo, la mujer toma prontamente LNG, lo que suprime la ovulación y por este mecanismo previene el embarazo. En la parte inferior se ilustra que después de tener una relación sexual en el período fértil del ciclo y muy cercano a la ovulación, la mujer toma el LNG, luego que ha ocurrido la ovulación y la fecundación. Tomado en este momento ya no interfiere con los procesos que siguen, la mujer experimenta atraso menstrual y se establece un embarazo clínicamente reconocible.

proceso en el cual adquieren movilidad hiperactivada, después de lo cual pierden en corto tiempo su vitalidad. Mientras se espera que ocurra la ovulación, la población de espermatozoides que se encuentra en la trompa de Fallopio está en constante renovación gracias a nuevos espermatozoides que llegan desde el reservorio cervical. Por lo tanto, para que la fecundación sea posible se requiere que la migración de nuevos espermatozoides desde el reservorio cervical hacia el sitio de fecundación persista hasta que ocurra la ovulación.

La eficiencia de este proceso se puede estimar observando la proporción de óvulos que se fecundan después de tener relaciones sexuales en el período fértil. Alvarez y cols (17) lograron recuperar el óvulo de la trompa de 20 mujeres de fertilidad probada, que tuvieron al menos una relación sexual en el período fértil verificada por el hallazgo de espermatozoides en el cuello uterino. Sólo 10 de estos óvulos estaban fecundados, lo cual indica que en las mejores condiciones, la tasa de fecundación in vivo en la especie humana es cercana al 50\%. Es decir, si 100 mujeres de fertilidad probada tienen relaciones sexuales no protegidas en el período fértil, en sólo 50 de ellas ocurriría la 
fecundación. Si es así en condiciones óptimas, es de suponer que en condiciones no óptimas, vale decir cuando el LNG está interfiriendo con la migración y función espermática, la tasa de fecundación está probablemente muy comprometida. La interferencia del LNG con la fase sostenida de la migración espermática, demostrada por Kesserü y cols (15), podría reducir o anular la probabilidad de que ocurra la fecundación, si es que llega a producirse la ovulación.

\section{EFECTOS DEL LNG SOBRE EL PROCESO OVULATORIO}

La posibilidad de que LNG administrado como $A E$ inhiba la ovulación en la mujer ha sido explorada por varios autores utilizando diversos diseños experimentales. En algunos casos, el LNG ha sido administrado en el día del pico de $\mathrm{LH}(\mathrm{LH}+0)$ en la orina, el cual es fácil de detectar, pero tiene el inconveniente de que la ovulación suele ocurrir en ese mismo día y por lo tanto es demasiado tarde para inhibirla. De hecho, los datos indican que dado el LNG en ese día, inhibe la ruptura folicular o interfiere con la formación de un cuerpo luteo sólo en una baja proporción de los ciclos tratados: a modo de ejemplo, 5 de 12 casos en el estudio de Hapangama y cols (18), y 0 de 11 casos en el estudio de Durand y cols (19). En algunos casos se intentó administrar el LNG dos días antes del pico de LH (LH-2) pero es difícil acertar y es casi imposible determinar en cuantos casos se logró. En el estudio de Marions y cols (20), el tratamiento con LNG en LH-2 suprimió el pico de LH en 5 de 5 casos, no se determinó si hubo o no hubo ruptura folicular, pero se detectó fase lutea de características normales en los 5 casos. Posteriormente perfeccionaron el diseño del estudio, agregando evaluación ecográfica del folículo, y confirmaron que en 7 de 7 casos el tratamiento con LNG suprimió la ovulación (21). En el estudio de Durand y cols, (19) el tratamiento dado presuntamente entre $\mathrm{LH}-2$ y LH-4 fue seguido de ruptura folicular y niveles significativamente disminuidos de progesterona en la fase lutea en 8 de 8 casos. Finalmente, en este mismo trabajo se trató a un grupo de mujeres en el día 10 del ciclo menstrual. En 12 de 15 se inhibió la ovulación (no hubo ruptura folicular ni fase lutea), y en otras tres se postergó la ovulación más allá del límite máximo de los 6 días que pueden esperar los espermatozoides al óvulo. Croxatto y cols (22) administraron LNG o placebo en la fase folicular cuando el folículo dominante tenía $12-14 \mathrm{~mm}, 15-17 \mathrm{~mm}$ o 18 $\mathrm{mm}$ de diámetro ( $\mathrm{n}=16-22$ por grupo) en un diseño cruzado, doble ciego y randomizado. Observaron muy buena correlación entre el diámetro folicular al momento de administración y la ausencia de ruptura folicular ecográfica y ausencia del pico preovulatorio de gonadotrofinas en los cinco días siguientes al tratamiento. Esto se observó en el $94 \%, 91 \%$ y $47 \%$ de los ciclos tratados con LNG, respectivamente, contra $62 \%, 45 \%$ y $13 \%$ en los ciclos controles de las mismas mujeres.

Es evidente que LNG administrado durante la fase folicular tiene la capacidad de interferir con el proceso ovulatorio, ya sea suprimiendo el pico de $\mathrm{LH}$, la ruptura folicular o la luteinización, lo cual parece depender del momento en que se administra. No se ha evaluado su posible interferencia con otros componentes críticos del proceso ovulatorio, como son la maduración del oocito y la expansión del cúmulo oósforo.

\section{EFECTOS SOBRE EL ENDOMETRIO}

Durand y cols (19), Marions y cols (20), y Palomino y cols (23) usaron distintos diseños para examinar la posibilidad de que el LNG interfiera con el desarrollo progestacional del endometrio, el cual es considerado esencial para que se implante el blastocisto. Para esto compararon aspectos morfológicos y moleculares en biopsias tomadas en el período receptivo luego de administrar LNG en el período periovulatorio. Encontraron mínimas o ninguna diferencia en los parámetros examinados en comparación con los ciclos controles de las mismas mujeres. Los resultados de estos estudios confirman que, cuando no suprime la fase lutea, el $L N G$ administrado como $A E$ no interfiere con el desarrollo progestacional del endometrio. Esto no es sorprendente ya que desde un punto de vista fisiológico y farmacológico, parece muy improbable que la administración de esta progestina altere el proceso que normalmente es inducido por la progesterona al actuar sobre el endometrio proliferativo. En conclusión, estos estudios, realizados en la mujer, muestran que LNG administrado en las dosis que se usan como $A E$ no produce cambios en el endometrio que sean suficientes para interferir con la implantación.

\section{ESTUDIOS EN LA RATA Y EN LA MONA}

La experimentación en animales permite examinar el efecto del LNG sobre etapas críticas del proceso reproductivo que no se pueden examinar en la mujer por limitaciones éticas y logísticas. 
Müller y cols (24) examinaron el efecto de LNG sobre la ovulación, la fecundación y la implantación en la rata. Para esto administraron LNG en distintos momentos del ciclo estral o de la preñez temprana. Observaron que LNG inhibió total o parcialmente la ovulación dependiendo del momento del tratamiento y de la dosis administrada, en tanto que las mismas dosis no interfirieron con la fecundación ni con la implantación. Por lo tanto, en la rata, la administración post-coital de LNG en dosis más altas que las usadas como $\mathrm{AE}$ en la mujer, y que son capaces de inhibir la ovulación, no interfieren con el desarrollo preimplantacional ni con la implantación.

Ortiz y cols (25) examinaron el efecto del LNG sobre la ovulación y sobre la tasa de embarazo en la mona Cebus apella. Para ello utilizaron un diseño en el cual cada hembra fue su propio control, siendo tratada con LNG en un ciclo y con placebo en otro, con un ciclo de descanso entremedio, y siendo la secuencia determinada en forma aleatoria. Observaron que la administración de LNG inhibió o postergó la ovulación cuando éste se administró en la fase folicular avanzada, mientras que no interfirió con la ovulación cuando esta era inminente. La administración postcoital de LNG, cuando se presumía que ya había ocurrido la fecundación, no disminuyó la tasa de implantación en comparación con los controles tratados con placebo.

En conclusión, el conjunto de resultados obtenidos en la mujer, en la rata y en la mona muestran que el efecto anticonceptivo de LNG administrado como $\mathrm{AE}$ se ejerce porque interfiere con procesos previos a la fecundación y no sustenta la hipótesis de que pueda prevenir embarazos interfiriendo con la implantación del embrión.

\section{BIBLIOGRAFÍA}

1. Piaggio $G$, von Hertzen $H$, Grimes DA, Van Look PF. On behalf of the Task Force on Postovulatory Methods of Fertility Regulation. Timing of emergency contraception with levonorgestrel or the Yuzpe regimen. Lancet 1999; 353: 721-4.

2. Piaggio $G$, von Hertzen $H$. Effect of the delay in the administration of levonorgestrel for emergency contraception. XVII FIGO Congress of Gynecology and Obstetrics, 2-7 november 2003, Santiago, Chile.

3. Espinós JJ. Contracepción postcoital: análisis crítico de la eficacia del método. 4ème congrès de la Société Européenne de Gynécologie. Barcelona, Octubre 2001.

4. Espinos JJ, Rodriguez-Espinosa J, Senosiain R,
Aura M, Vanrell C, Gispert M, Vega C, y cols. The role of matching menstrual data with hormonal measurements in evaluating effectiveness of postcoital contraception. Contraception 1999; 60: 243-7

5. Espinós JJ. Emergency Contraception: Evaluation of Effectiveness. XVII FIGO Congress of Gynecology and Obstetrics, 2-7 November 2003, Santiago, Chile.

6. Raymond E, Taylor D, Trussel J. Steiner MJ. Minimum effectiveness of the levonorgestrel regimen of contraception. Contraception 2004; 69: 79-81.

7. Wilcox AJ, Weinberg CR, Baird DD. Post ovulatory ageing of the oocyte and embryo failure. Human Reproduction 1998; 13:394-7.

8. Senosiain R, Matas A, Vanrell C, Peon S, Mazzanti I, Gou M, Gonzalez M, y cols. ¿Cuál es el grado de exposición seminal en las parejas que solicitan contracepción postcoital? 4ème congrès de la Société Européenne de Gynécologie. Barcelona, Octubre 2001.

9. Croxatto HB, Ortiz ME, Müller AL. Mechanism of action of emergency contraception. Steroids 2003; 68: 1095-8.

10. Gemzell-Danielsson K, Marions L. Mechanism of action of mifepristone and levonorgestrel when used for emergency contraception. Human Reproduction Update, 2004; 10: 341-8.

11. Yeung WS, Chiu $P C$, Wang $\mathrm{CH}$, Yao $Y Q$, Ho $P C$. The effects of levonorgestrel on various sperm functions. Contraception 2002; 66: 453-7.

12. Bahamondes L, Nascimento JAA, Munuce MJ, Fazano $\mathrm{F}$, Faúndes $\mathrm{A}$. The in vitro effect of levonorgestrel on the acrosome reaction of human spermatozoa from fertile men. Contraception 2003; 68: 559.

13. Brache V, Faúndes A, Johansson E, Alvarez F. Anovulation, inadequate luteal phase and poor sperm penetration in cervical mucus during prolonged use of Norplant ${ }^{\circledR}$ implants. Contraception 1985; 31: 261-73.

14. Croxatto HB, Diaz S, Salvatierra AM, Morales P, Ebensperger C, Brandeis A. Treatment with Norplant ${ }^{\circledR}$ subdermal implants inhibits sperm penetration through cervical mucus in vitro. Contraception 1987; 36: 193-201.

15. Kesserü E, Garmendia F, Westphal M, Parada J. The hormonal and peripheral effects of d-norgestrel in postcoital contraception. Contraception 1974; 10: 411-24.

16. Croxatto H.B. Gamete Transport. Cap. 18. En: Adashi EY, Rock JA, Rosenwaks Z. (eds): Reproductive Endocrinology, Surgery, and Technology. New York, USA: Lippincot-Raven, 1996; 386-402.

17. Alvarez F, Brache V, Fernandez E, Guerrero B, Guiloff E, Hess R, Salvatierra AM, y cols. New insights on the mode of action of intrauterine contraception devices in women. Fertil Steril 1988; 49: 768-73.

18. Hapangama D, Glasier AF, Baird, DT. The effects of 
pre-ovulatory administration of levonorgestrel on the menstrual cycle. Contraception 2001; 63: 123-9.

19. Durand M, Cravioto MC, Raymond EG, DuranSanchez O, Cruz-Hinojosa ML, Castell-Rodriguez A, Schiavon R, y cols: On the mechanisms of action of short-term levonorgestrel administration in emergency contraception. Contraception 2001; 64: 227-34.

20. Marions L, Hultenby K, Lindell I, Sun X, Stabi B, Gemzell Danielsson K. Emergency contraception with mifepristone and levonorgestrel: mechanism of action. Obstet Gynecol 2002; 100: 65-71.

21. Marions L, Cekan SZ, Bygdeman M, GemzellDanielsson K. Effect of emergency contraception with levonorgestrel or mifepristone on ovarian function. Contraception 2004; 69: 373-7.

22. Croxatto HB, Brache V, Pavez M, Cochon L, Forcelledo ML, Massai R, Faundes A, y cols. Pituitary-ovarian function following the standard levonorgestrel emergency contraceptive dose or a single $0.75 \mathrm{mg}$ dose given on the days preceding ovulation. Contraception 2004, en prensa.

23. Palomino A, Boric A, Gabler F, Espinoza A, Vega $M$, Devoto L. Efecto de levonorgestrel como anticonceptivo de emergencia sobre receptores de progesterona durante la ventana de implantación. Revista Cubana de Salud Pública 2003; 29 Supl № 1: 38.

24. Müller AL, Llados CM, Croxatto HB. Postcoital treatment with levonorgestrel does not disrupt postfertilization events in the rat. Contraception 2003; 67: 415-9.

25. Ortiz ME, Ortiz RE, Fuentes $M$, Parraguez VH, Croxatto HB. Post-coital administration of levonorgestrel does not interfere with post-fertilization events in the new-world monkey Cebus apella. Human Reproduction 2004; 19:1352-6. 